Portland State University

PDXScholar

Chemistry Faculty Publications and

Presentations

Chemistry

8-1-1983

\title{
Generalized response of chemiluminescence analyzers
}

\author{
A. A. Mehrabzadeh \\ Portland State University \\ Robert J. O'Brien \\ Portland State University \\ Thomas M. Hard \\ Portland State University
}

Follow this and additional works at: https://pdxscholar.library.pdx.edu/chem_fac

Part of the Analytical Chemistry Commons

Let us know how access to this document benefits you.

\section{Citation Details}

Mehrabzadeh, A. A., O'Brien, R. J., \& Hard, T. M. (1983). Generalized response of chemiluminescence analyzers. Review Of Scientific Instruments, 54(12), 1712-1718.

This Article is brought to you for free and open access. It has been accepted for inclusion in Chemistry Faculty Publications and Presentations by an authorized administrator of PDXScholar. Please contact us if we can make this document more accessible: pdxscholar@pdx.edu. 


\title{
Generalized response of chemiluminescence analyzers
}

\author{
A. A. Mehrabzadeh, R. J. O'Brien, and T. M. Hard \\ Chemistry Department and Environmental Sciences Doctoral Program, Portland State University, Portland, \\ Oregon 97207
}

(Received 7 June 1983; accepted for publication 5 August 1983)

\begin{abstract}
The mass flow and chemical kinetic equations for a gaseous chemiluminescence (CL) analyzer are formulated and solved. The resultant equation can be used to predict the absolute response of the analyzer as a function of the sample flow rate, the sample gas pressure, the chamber pressure, the chamber volume, the mass flow rate and mole fraction of the reagent gas, and the rate constants of the relevant chemical processes. Thus, the equation allows optimization of these parameters. It is shown that for varying sample pressure the analyzer can be used to measure either concentration or mole fraction and that interfering reactions can sometimes be discriminated against by chamber pressure variation. The equations apply equally well to a flowing-liquid-phase CL analyzer, if the chemical mechanism considered is appropriate.
\end{abstract}

PACS numbers: $82.40 . T c, 78.60 . P s, 51.70 .+\mathrm{f}$

\section{INTRODUCTION}

Chemiluminescence (CL) is an optical phenomenon which is widely used for analytical purposes. Many laboratory-developed CL analyzers have been described in the literature, and commercial versions are available. The technique has been extensively applied to gaseous measurements of ozone, nitric oxide, and some other species. Recently, liquid phase CL has seen increasing development.

The basic chemical mechanism for the generation of a chemiluminescent intermediate is fairly well understood in several cases. ${ }^{1-3}$ The reagent gas (generally in high concentration) reacts with a detected trace gas to produce an excited (usually electronic) state. This state then emits radiation which is detected by a photomultiplier. The mechanism is complicated by side reactions which produce nonemitting products and quenching of the chemiluminescence, both of which reduce the response. Quenching may seem to make it advantageous to operate the detection chamber at reduced pressure, and some instruments are operated at pressures of several torr. Background and interference come from ambient light and from other chemiluminescent reactions. The detection limit is set by the dark current of the photomultiplier, the interference and background, and the inherent kinetic processes of the detection and reagent gases.

Since the first development of a homogeneous gas phase CL analyzer, ${ }^{4}$ many papers utilizing this technique have appeared (e.g., Refs. 5-20; reviews Refs. 21,25). The basic equation governing maximum response has been used by several authors, ${ }^{10,16,18}$ and the general equation for plug-flow response has been obtained by Steffenson and Stedman, ${ }^{11}$ and discussed by Ridley. ${ }^{21}$ The requirements for achieving maximum response and the optimization considerations for the various flows and pressures in a CL analyzer have been treated in part by various authors. However, no general, systematic treatment has appeared.

In practice, the response of a CL analyzer can be partially optimized by empirical adjustment of pressures and flows. However, full optimization involves a choice of the chamber volume and the type of pump, not so easily varied. Furthermore, optimization in a particular situation may not necessarily involve achievement of maximum response, a situation more difficult to handle empirically. Here, we have obtained a general solution to the kinetic equations for a $\mathrm{CL}$ analyzer and optimized it for all relevant parameters under two distinct operating modes. The behavior of a CL analyzer under nonmaximum response conditions is discussed and shown to be advantageous in some circumstances.

\section{THEORETICAL APPROACH}

\section{A. Exponential-dilution reactor}

The simplest mechanism for a chemiluminescent process is the following (the $k$ 's above the arrows are the kinetic rate constants):

$$
\begin{aligned}
& R+D^{k_{t}} \rightarrow E, \\
& R+D \stackrel{k_{t_{t}}}{\rightarrow} \text { loss, } \\
& E \stackrel{k_{f}}{\rightarrow} h v \text { fluorescence, } \\
& E+M \stackrel{k_{q}}{\rightarrow} \text { quenching, }
\end{aligned}
$$

here, $R$ is the reagent gas, $D$ is the trace gas to be detected, $E$ is an excited state product of the reaction between $R$ and $D$, and $M$ is any quenching gas, usually air and the reagent. To these chemical processes must be added the flow equations which determine the response of the analyzer. Steffenson and Stedman ${ }^{11}$ have obtained the general response equation of a plug-flow CL analyzer, but have not treated the characteristics of the equation in detail. We have found that detailed optimization of the plug-flow response with respect to reagent flow leads to an equation which must be solved numerically. Furthermore, although Steffenson and Stedman argued against large-volume reactors because of light collection difficulties, we believe that large volumes are often desirable. Thus we treat first the case of an exponential-dilution reactor, since it seems most applicable to a large chamber. For this situation the flow processes are the following: 


$$
\begin{aligned}
& \rightarrow D \text { flow rate }=F_{D} \mathrm{cc} / \mathrm{s}, \\
& \rightarrow R \text { flow rate }=F_{R} \mathrm{cc} / \mathrm{s},
\end{aligned}
$$

everything $\rightarrow$ pump flow rate $=F_{M} \mathrm{cc} / \mathrm{s}$.

A detector is illustrated schematically in Fig. 1. The large reaction volume is viewed by the photomultiplier using a chamber with reflective walls. The three valves are used to control relative sample and reagent flow rates and total pressure in the chamber. One or more valves may be wide open or eliminated.

Processes (5) and (6) may be thought of as zero-order reactions whose molecular flows are given by the appropriate flow rate of the sample or reagent stream times the concentration of the species in the incoming stream $\left([D]_{0}\right.$ or $\left.[R]_{0}\right)$. Process (7) is exponential dilution for a well-mixed reactor. Kinetic equations may be written for each species and set equal to zero for steady emission in a well-mixed reaction chamber of volume $V$. These equations, expressed in terms of molecules/s, are as follows:

$V \frac{d[D]}{d t}=F_{D}[D]_{0}-\left(k_{E}+k_{L}\right)[R][D] V-F_{M}[D]$

$V \frac{d[R]}{d t}=F_{R}[R]-\left(k_{E}+k_{L}\right)[R][D] V-F_{M}[R]$,

$$
\begin{aligned}
V \frac{d[E]}{d t}= & k_{E}[R][D] V-k_{q}[E][M] V \\
& -k_{f}[E] V-F_{M}[E] .
\end{aligned}
$$

It is desired to solve for the concentration of the emitting species $E$, since $k_{f}[E] V$ is the chemiluminescence in photons. The last term in Eq. (10) can be neglected whenever the lifetime of $E$ is much shorter than its residence time in the chamber. Thus,

$$
[E]=k_{E}[R][D] /\left(k_{f}+k_{q}[M]\right) \text {. }
$$

In general, of course, reaction (4) will have various rate constants depending upon the identity of the quenching gas, $M$. Thus, the term $k_{q}[M]$ represents a sum over all significant quenchers: $k_{q}[M]=\Sigma_{i} k_{q i} X_{i}[M]$, where $X_{i}$ is the mole fraction of species $i$ in the chamber.

Linear instrumental response requires $[R]_{0} \gg[D]_{0}$, so $[R]$ is governed only by flow processes and the second term in Eq. (9) is negligible. Mass conservation requires

$$
F_{M}[M]=F_{D}\left[P_{D}\right]+F_{R}\left[P_{R}\right] \text {. }
$$

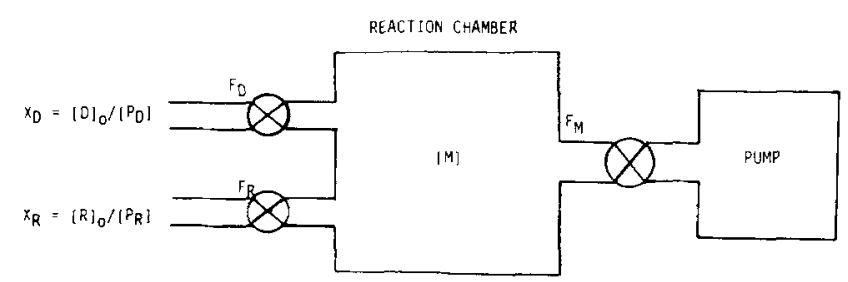

FIG. 1. Schematic chemiluminescence cell. Reagent gas, at mole fraction $X_{R}$, enters with flow $F_{R}$, and may be pure or diluted in a carrier at a total pressure $\left[P_{R}\right]$. The gas to be detected, at concentration $[D]_{0}$, in air of ambient pressure $\left[P_{D}\right]$, enters with flow $F_{D}$. Chamber pressure is $[M]$ and exit flow rate is $F_{M}$. The inlet and exit flows and the chamber pressure are controlled by the three valves, any of which may be fully open.
Here, $\left[P_{D}\right]$ is the ambient total pressure that the detector samples, $\left[P_{R}\right]$ is the total pressure of the reagent stream before entering the chamber, and $[M]$ is the total pressure inside the reaction chamber. For convenience we here refer to an actual concentration as a "pressure" in units of molecules/cc. Flows are in cc/s at the respective pressures, $\left[P_{D}\right]$, $\left[P_{R}\right]$, and $[M]$.

We define the concentration response of the analyzer (in photons $\mathrm{s}^{-1} /$ molecule $\mathrm{cm}^{-3}$ ambient) as $r=k_{f}[E] V /[D]_{0}$. The response may then be obtained from Eqs. (8)-(12) and is a function of the kinetic parameters $V$ and $X_{R}$ and of the flow parameters $F_{D}, F_{M}, F_{R}\left[P_{R}\right]$, and $[M]$. Among the latter four parameters, mass conservation requires that one variable be dependent. Mass conservation has apparently not been considered explicitly in previous treatments of $\mathrm{CL}$ analyzers. Nevertheless, it places fundamental constraints on the response of an analyzer as a function of the flow parameters. For instance, an analyzer is inherently sensitive to the chamber pressure, $[M]$. Assuming that the reagent flow is constant, chamber pressure may be decreased either by increasing $F_{M}$ or by decreasing $F_{D}$. Our approach is to consider three types of response limitation: by $F_{M}, F_{D}$, and $F_{R}$. The first limit applies to many analyzers which have operated in the upper atmosphere $(10,15,16,18)$; the second to many analyzers such as commercial $\mathrm{NO}_{x}$ analyzers used in monitoring pollutants at ground level; while the third apparently has not been properly exploited before.

Solution of Eqs. (8)-(12) results in the following response equation for an exponential-dilution (ed) $\mathrm{CL}$ analyzer:

$$
\begin{aligned}
r_{\mathrm{ed}}= & \frac{1}{\left[P_{D}\right]}\left(\frac{1}{\frac{k_{q}}{k_{f}}+\frac{1}{[M]}}\right) \\
& \times\left(\frac{1+Z}{Y F_{M}}+\frac{2+Z+1 / Z}{k_{E} V X_{R}[M]}\right),
\end{aligned}
$$

or

$r_{\mathrm{ed}}=\left(\frac{k_{f}}{k_{f}+k_{q}[M]}\right)\left(\frac{1}{\frac{1}{Y F_{D}}+\frac{\left[P_{D}\right](2+Z+1 / Z)}{k_{E} V X_{R}[M]^{2}}}\right)$,

where $X_{R}$ is the mole fraction of $R$ in the reagent stream, and the yield of excited intermediate is $Y=k_{E} /\left(k_{E}+k_{L}\right)$. The relative inlet total molecular flow ratio $Z$ is defined as

$$
Z=\frac{F_{R}\left[P_{R}\right]}{F_{D}\left[P_{D}\right]}
$$

The inlet flows appear only as their ratio in Eq. (13), and this simplifies the optimization of the response with respect to inlet flows. Although previous workers have discussed reagent flow sufficient to react $95 \%$ of $D$, proper optimization of reagent has not been carried out.

In Eq. (13), the response is a function of $[M], Z$, and either $F_{M}$ or $F_{D}$. The first choice of dependent variables illustrates a double dependence upon $[M]$. Equation (13a) contains the product of two expressions, each having the form of the resultant of two conductances connected in series, $C=1 /\left(1 / C_{1}+1 / C_{2}\right)$. Each of these expressions ap- 
proaches the behavior of whichever conductance is smaller. In the first expression, the conductances are the quenching half-pressure $k_{f} / k_{q}$ and the chamber pressure [M]. In the second expression the conductances are functions of the intermediate's formation rate and its residence time within the chamber. Thus the limiting behavior with respect to $[M]$ can be found by inspection.

\section{Mode 1: Maximum response limited by $F_{M}$}

The first operational mode applies to an analyzer with constant $F_{M}$, whose chamber pressure is controlled by variation of the entering flow rates. We first determine the optimum value of $Z$ by zeroing the partial derivative of Eq. (13a) with respect to $Z$ at constant $F_{M}$ and $[M]$. This results in the following condition for optimum relative inlet molecular flows:

$$
Z_{\text {ed }}^{\mathrm{opt}}=\left(1+\left(k_{E}+k_{L}\right) V X_{R}[M] / F_{M}\right)^{-1 / 2} .
$$

For $[M] \ll F_{M} /\left(k_{E}+k_{L}\right) V X_{R}$, this reduces to equal inlet molecular flows. At high chamber pressure, however, relatively less reagent flow is required to achieve maximum response. This situation is one where $F_{M}$, the sum of $F_{D}\left[P_{D}\right]+F_{R}\left[P_{R}\right]$ and, hence, $[M]$ remain constant and only the ratio $Z$ of inlet molecular flows varies. The behavior of Eq. (13) under these conditions is illustrated in Fig. 2. A series of curves is drawn, each one for constant $[M]$, as a function of flow ratio $Z$. As $[M]$ increases, the response increases toward an asymptote described next and the position of the maximum moves toward less reagent flow.

The asymptotic behavior with chamber pressure $[M]$ is described by considering the high-pressure limiting case of Eq. (13). This gives the maximum achievable response $r_{\max }$ when there are no restrictions on sample or reagent flows other than the pumping speed of the pump, $F_{M}$ :

$$
r_{\max }=\frac{k_{f} Y F_{D}}{k_{q}[M]}=\frac{k_{f} Y F_{M}}{k_{q}\left[P_{D}\right](1+Z)} .
$$

At sufficiently high [ $M$ ], the optimal $Z$ becomes much less than 1. Formulas similar to Eq. (15) have been derived for the $\mathrm{NO} / \mathrm{O}_{3}$ analyzer. ${ }^{10,16,18}$ However, the first form, expressed in terms of $F_{D}$ and [M], may be subject to misinterpretation-for instance if it leads to the general conclusion that the signal depends upon $F_{D}$ or that higher response may be obtained by reducing $[M]$.

Figure 3 shows the asymptotic behavior of Eq. (13a) more clearly. Consider first the solid curves drawn for $\left[P_{D}\right]=1 \mathrm{~atm}, F_{M}=1000 \mathrm{cc} / \mathrm{s}$ and $Z=1$. In this family of curves, the dependence of response on sample inlet flow $\left(F_{D}\right)$ is shown for various constant values of $k_{E} V X_{R}$. Varying inlet flow at constant $Z$ and $F_{M}$ of course produces a proportional variation in chamber pressure $[M]$. As $F_{D}, F_{R}$, and $[M]$ increase, the response also increases toward a limiting value given by the right-hand form of Eq. (15). This asymptote is the plateau region noted for plug flow by Steffenson and Stedman. ${ }^{11}$ The plateau as discussed by these authors and as illustrated in Fig. 3 is not quite a maximum with respect to $Z$, since a further increase by almost a factor of 2 in response could be achieved for the solid curves by a decrease of $Z$ from 1 to $Z$ opt .

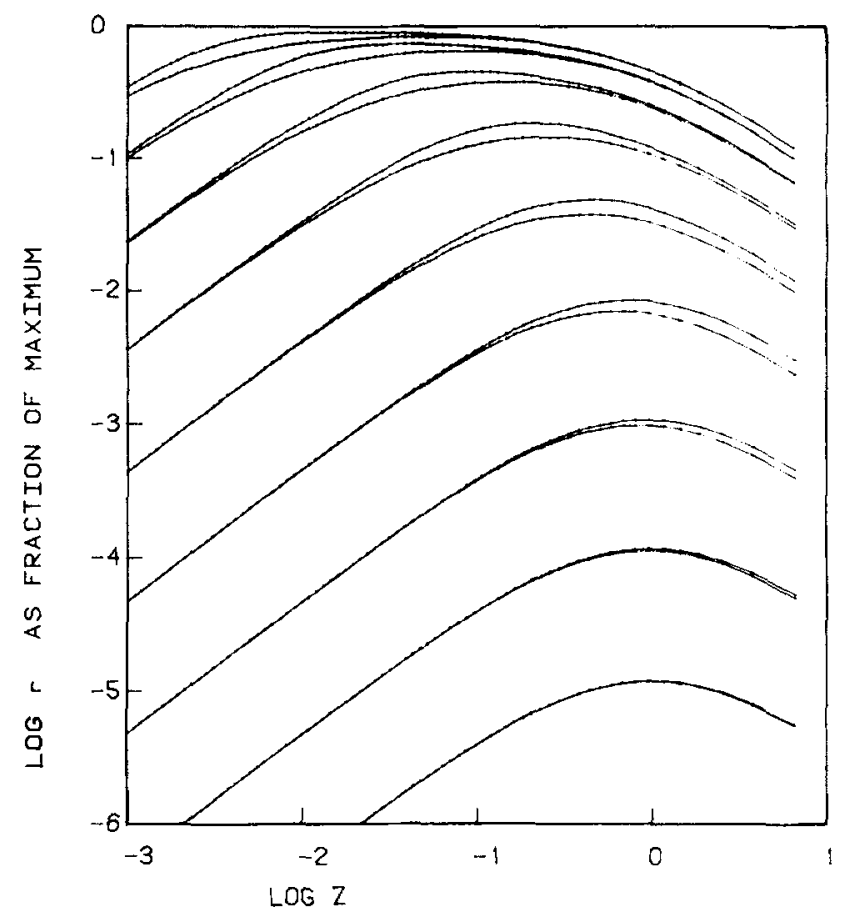

FIG. 2. Dependence of analyzer response upon relative flow rates at the inlet ports according to Eq. (13). All parameters are held constant except reagent gas and detection gas total molecular flows, whose ratio $Z$ is varied while their sum is constant. Chamber pressures increase upward from $0.001-10$ atm, in steps of $10^{1 / 2}$. The maxima agree with Eqs. (14) and (20). The plugflow curves coincide with or lie slightly above the corresponding exponential-dilution curves. Here, $F_{M}=1000 \mathrm{cc} / \mathrm{s}, k_{E} V X_{R}=2 \times 10^{-24} \mathrm{~cm}^{h}$ molec ${ }^{-1} s^{-1}$.

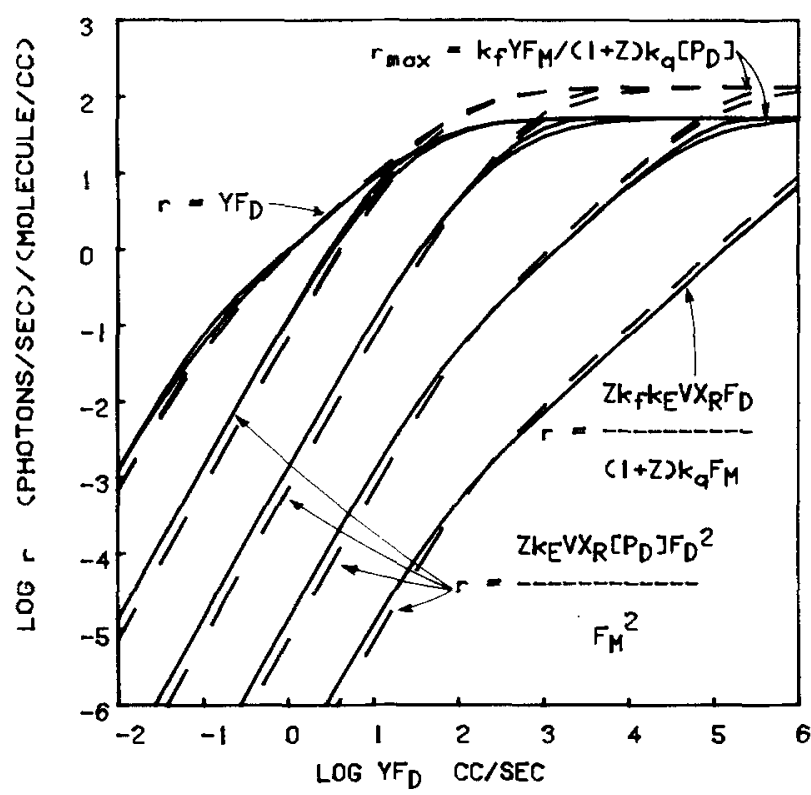

FIG. 3. Response of a Mode $1 \mathrm{CL}$ analyzer for varying sample flow rate $F_{D}$ at constant $F_{M}$ and $Z$. As $F_{D}$ increases, the response increases toward an asymptote. The solid curves use ambient pressure $\left[P_{D}\right]=1 \mathrm{~atm}, Z=1$, and several values of $k_{E} V X_{R}$; the dashed curves are for $\left[P_{D}\right]=0.1 \mathrm{~atm}, Z=10$. All curves: $k_{f} / k_{q}=2 \times 10^{17}$ molec $/ c c, F_{M}=1000 \mathrm{cc} / \mathrm{s}$. Upper curves: $k_{E} V X_{R}=1 \times 10^{-10} \mathrm{~cm}^{6} \mathrm{molec}^{-1} \mathrm{~s}^{-1}$, decreased by a factor of 100 for each successive lower curve. Plug-flow responses coincide with or lie slightly above those for exponential dilution. 
In the high-flow limit of Fig. 3, the response is independent of the mole fraction of the reagent gas, as well as independent of the chamber pressure and volume. This limit is reached when the two conductance expressions in Eq. (13a) reach their maximum values at a pressure given by

$$
[M]>(1+Z) F_{M} / Z\left(k_{E}+k_{L}\right) V X_{R},
$$

and

$$
[M]>k_{f} / k_{q} \text {. }
$$

Condition $(16 \mathrm{~b})$ is contrary to what would be expected from examining quenching behavior alone, and indicates that efficient fluorescence of the intermediate is less important than high molecular throughput and adequate residence time. Similar inequalities can be obtained for $F_{D}$ using Eq. (13b).

Since the maximum signal is proportional to $F_{M}$, it is usually desirable to increase the chamber volume, reagent mole fraction, and/or the chamber pressure as necessary to satisfy Eq. (16) and reach the plateau of maximum response.

The dependence of response upon sample pressure $\left[P_{D}\right]$ is illustrated in Fig. 3 by the dashed curves which are drawn with $\left[P_{D}\right]=0.1 \mathrm{~atm}$ and $Z=10$. The two families of curves simulate a situation where atmospheric pressure drops by an order of magnitude, but $F_{D}$ and $F_{R}\left[P_{R}\right]$ remain constant due to choked inlet flow. Thus $Z$ increases by 10 . Here, as in the solid curves, more response could be achieved by decreasing $Z$ to $Z^{\text {opt }}$. Here the potential gain is about a factor of 10 . Note that in Fig. 3 the dashed curves indicate lower response at low $F_{D}$ but higher response at high $F_{D}$ for $\left[P_{D}\right]=0.1 \mathrm{vs}$ $1.0 \mathrm{~atm}$.

The dependence of $r$ upon sampled pressure $\left[P_{D}\right]$ is significant since $C L$ analyzers are often found to be sensitive to ambient pressure. This can be a disadvantage unless the pressure dependence is understood. In the plateau region described by Eq. (15), the limiting absolute response $r_{\max }$ is inversely proportional to ambient pressure $\left[P_{D}\right]$. This means that if ambient pressure is reduced and the mole fraction, $[D]_{0} /\left[P_{D}\right]$, remains constant, the detector will give a constant signal $r[D]_{0}$. This potential mole fraction response is advantageous for determining altitude profiles of atmospheric species because it means the absolute sensitivity increases with altitude. The mole fraction response in units of photons $\mathrm{s}^{-1} /$ unit mole fraction ambient is given by $r\left[P_{D}\right]$ with $\left[P_{D}\right]$ in concentration units. To achieve this constant response, it is necessary that $F_{M}$ remain constant. (In general $F_{M}$ will vary with $[M]$ and/or $\left[P_{D}\right]$, depending upon the pump's operating characteristics, but we ignore this for simplicity). If $F_{M}$ is constant, then variation of chamber pressure $[M]$ with altitude is immaterial as long as the detector remains on the response plateau. In order to be sure of remaining on the plateau and, hence, realizing the constant mole fraction response, the inequalities of Eq. (16) must be interpreted in terms of the lowest pressures to be encountered, and $Z$ should be small enough that its variation with $\left[P_{D}\right]$ is not significant.

In the low-pressure $[M]$ or low-flow $F_{D}$ portion of Fig. 3 , the response curves are given by

$$
r \rightarrow \frac{k_{E} V X_{R}[M]^{2}}{(2+Z+1 / Z)\left[P_{D}\right]}=\frac{Z k_{E} V X_{R} F_{D}^{2}\left[P_{D}\right]}{F_{M}^{2}} .
$$

The response in this region is proportional to chamber volume, the rate constant to produce $E$, the reagent gas mole fraction, and the square of the chamber pressure. The quadratic dependence is shown by the slope of 2 in the steepest portions of the curves in Fig. 3. The first expression for $r$ in Eq. (17) is appropriate to an analyzer in which the chamber pressure is independent of ambient pressure. Such independence could only be achieved by external flow control of $F_{D}$. Practical chamber inlets and pumps are more likely to have constant $F_{D} / F_{M}$ and, hence, $[M]$ proportional to $\left[P_{D}\right]$. In the latter case the response to constant absolute concentration is proportional to $\left[P_{D}\right]$, and the constant mole fraction signal is proportional to $\left[P_{D}\right]^{2}$. This is obviously undesirable for situations where ambient pressure may change. Thus the operation of analyzers off the plateau region may not be satisfactory for obtaining altitude profiles of atmospheric gases, or even for precise concentration measurements under conditions of slight pressure variation.

Besides the two pressure extremes, there are crossover regions of slope $=1$ in Fig. 3. These occur where either condition (16a) or (16b) is satisfied, but not the other. When only (16a) is satisfied, the response is proportional to absolute concentration $[D]_{0}$, and independent of ambient pressure. The uppermost pair of curves coincides in response for a wide range of values of $F_{D}$. Since only condition (16a) is satisfied Eq. (13) becomes $r=Y F_{M}[M] /(1+Z)\left[P_{D}\right]$ $=Y F_{D}$. Thus independence of the concentration response upon ambient pressure can be achieved by choosing the correct values of $V, X_{R}$, and [ $\left.M\right]$. Other regions of slope $=1$, to the lower right in Fig. 3, correspond to satisfying only condition(16b), giving $r=Z k_{f} k_{E} V X_{R} F_{D} /(1+Z) k_{q} F_{M}$, where $Z$ varies with ambient pressure.

\section{Mode 2: Sample $\left(F_{D}\right)$ limited}

Under some circumstances it may be desirable to limit the value of $F_{D}$-for instance when the sampled volume is limited. The distinctness of this situation relative to the response equation has been mentioned by Steffenson and Stedman, ${ }^{11}$ but has not been previously treated, although some commercial instruments operate in this mode. Assuming $F_{D}$ is fixed by flow or other constraints so that the plateau for a given pumping speed $F_{M}$ cannot be reached, then maximum response occurs at a nonasymptotic value of $[M]$ which may be quite different from $\left[P_{D}\right]$. Starting with Eq. (13b), zeroing the partial derivative of $r$ with respect to $Z$ yields an optimal value of $Z_{\text {ed }}^{\text {opt }}=1$, or equal inlet molecular flows. Optimal chamber pressure is found by setting the partial derivative of $r$ with respect to $[M]$ equal to zero, and solving the resultant cubic equation, which gives

$$
[M]=\left(-q / 2+s^{1 / 2}\right)^{1 / 3}+\left(-q / 2-s^{1 / 2}\right)^{1 / 3},
$$

where

$$
\begin{aligned}
& s=(p / 3)^{3}+(q / 2)^{2} ; \\
& q=-8 k_{f} Y F_{D}\left[P_{D}\right] / k_{E} k_{q} V X_{R} ; \\
& p=-4 Y\left[P_{D}\right] / k_{E} V X_{R} .
\end{aligned}
$$

The behavior of Eq. (13b) is illustrated for two values of $k_{E} V X_{R}$ and for several constant values of $Y F_{D}$ in Fig. 4. 


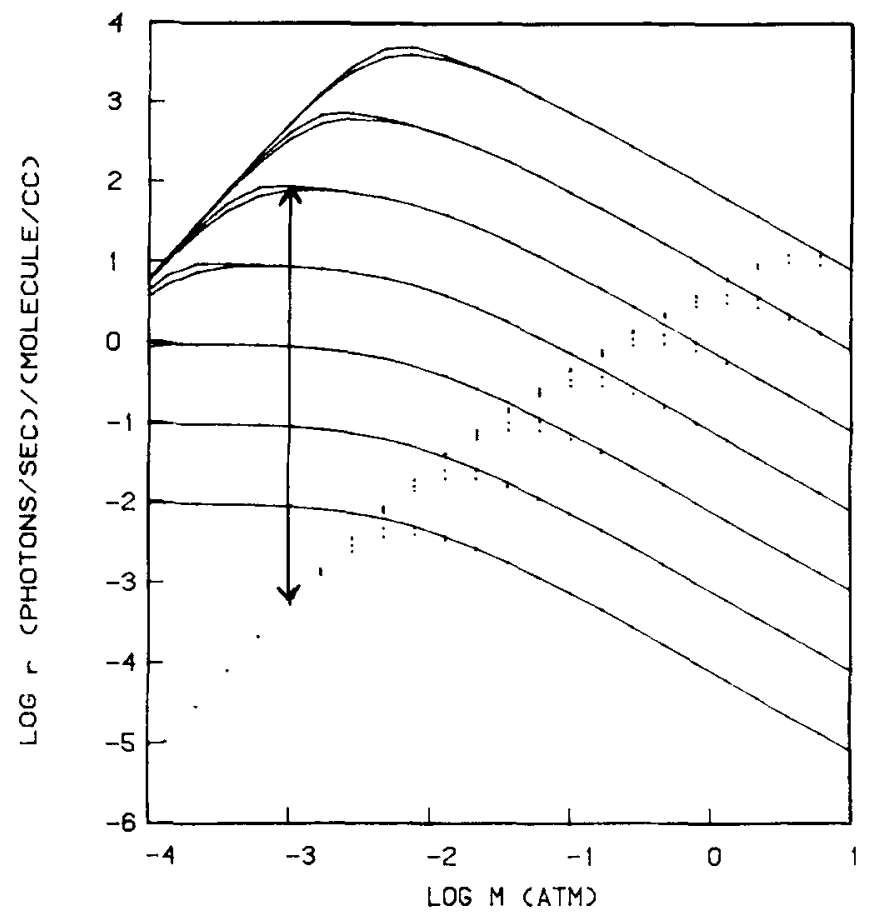

Fig. 4. Response of a Mode 2 analyzer vs chamber pressure [ $M$ ] at several constant sample flow rates $F_{D}$. Each curve shows a maximum at a value of $[M]$ given by $\mathrm{Eq}$. (18), in contrast with the asymptotic responses of Fig. 3 . Solid curves are for $k_{E} V X_{R}=1 \times 10^{-10}$, dotted curves for $1 \times 10^{-16} \mathrm{~cm}^{6}$ molec $^{-1} \mathrm{~s}^{-1} . F_{D}$ increases by successive factors of 10 from the lowest curves, from $F_{D}=0.01$ to $10000 \mathrm{~cm}^{3} \mathrm{~s}^{-1}$. Discrimination against a slow reaction is indicated by the two-headed arrow.

From the figure it is seen that for higher values of $k_{E} V X_{R}$ it is desirable to increase $F_{M}$ at constant $F_{D}$ in order to reduce the chamber pressure and obtain a maximum signal. Operation in this mode has the disadvantage that the response may be sensitive to ambient pressure.

\section{Mode 3: Reagent $\left(F_{R}\left[P_{R}\right]\right)$ limited}

The third operational mode has not been previously recognized. However, it has the advantage of saving signifcantly on reagent consumption and achieving insensitivity of response to variations in ambient (i.e., chamber) pressure. This is done at a minor sacrifice in response.

In the limit of small $Z$ (while still requiring $[R] \gg[D]$ ), the response Eq. (13a) becomes

$$
\boldsymbol{r} \rightarrow \boldsymbol{Z} k_{E} k_{f} \boldsymbol{V} X_{R}[M]^{2} /\left(k_{f}+k_{q}[M]\right)\left[P_{D}\right] .
$$

If Eq. $(17 \mathrm{~b})$ is satisfied, the mole fraction response

$$
r\left[P_{D}\right] \rightarrow F_{R}\left[P_{R}\right] k_{f} k_{E} V X_{R}[M] / k_{q} F_{D}\left[P_{D}\right]
$$

and remains constant as long as $[M]$ is proportional to $\left[P_{D}\right]$. On the other hand, if the reverse of Eq. (16b) is satisfied, the concentration response

$$
r \rightarrow F_{R}\left[P_{R}\right] k_{E} V X_{R}[M]^{2} / F_{D}\left[P_{D}\right]^{2},
$$

and this response also remains constant as long as $[M]$ is proportional to $\left[P_{D}\right]$. The latter condition is a special case of the linear concentration response region discussed under Mode 1. Thus it is possible to operate off the response pla- teau and still retain insensitivity to variation in ambient pressure.

\section{B. Third-order reactions}

Other possible mechanisms involve pressure dependence of Reactions 1 and 2 . If both of these involve a third body $M$, then the yield term remains the same. If reaction 1 is pressure-dependent, then $k_{E}[M]$ replaces $k_{E}$ in Eqs. (13) and (17), and the response varies as $[M]^{3}$ in the low-pressure limit. In this case Eq. (18) must be rederived and the optimum chamber pressure for constant $F_{D}$ will be higher.

\section{Plug-flow reactor}

As discussed above, large reactor volumes are often desirable in order to achieve the plateau of maximum response. In this case it is likely that an exponential-dilution chamber would approach the actual experimental situation most closely. However, for small reactor volumes, a plug-flow design might be preferable, since reacting gases are not diluted with the exhausted reaction mixture. Solution of the kinetic equations indicates that the improvement is small for the range of values illustrated in Figs. $2-4$. Starting with Eq. (9) of Steffenson and Stedman, ${ }^{11}$ the basic dependence of response upon $F_{M}$ rather than $F_{D}$ for a Mode 1 plug-flow (pf) analyzer can be shown by substituting expressions in $Z$ for the two inlet flows to yield

$$
\begin{aligned}
r_{\mathrm{pf}}= & \frac{Y F_{M}[M]}{(1+Z)\left(1+k_{q}[M] / k_{f}\right)\left[P_{D}\right]} \\
& \times\left(1-\exp \frac{-Z\left(k_{E}+k_{L}\right) V X_{R}[M]}{(1+Z) F_{M}}\right) .
\end{aligned}
$$

Steffenson and Stedman point out that the exponent is the ratio of the chamber residence time of $D$ to its chemical lifetime. This equation reduces, as it must, to Eq. (15) in the limit of large reactor volume $V$ and high $[M]$. Steffenson and Stedman optimized reagent flow empirically for their analyzer for a single experimental condition and then retained this value in all experiments. However, a general optimum flow condition is found by zeroing the partial derivative of Eq. (19) with respect to $Z$ and is given implicitly by

$$
\begin{aligned}
Z_{\mathrm{pf}}^{\mathrm{opt}}= & \frac{\left(1+Z_{\mathrm{pf}}^{\mathrm{opt}}\right)}{\left(k_{E}+k_{L}\right) V X_{R}[M]} \\
& \times \ln \left(1+\frac{\left(k_{E}+k_{L}\right) V X_{R}[M]}{\left(1+Z_{\mathrm{pf}}^{\mathrm{opt}}\right)}\right) .
\end{aligned}
$$

At small volume or low pressure this reduces to $Z_{\mathrm{pf}}^{\mathrm{opt}}=1$, as for exponential dilution. However, at high $[M]$ the maximum occurs at even lower values of reagent flow than was the case for exponential dilution, as illustrated in Fig. 2. Unfortunately, under nonlimiting conditions, Eq. (20) must be solved numerically. Plug-flow response is also plotted in Figs. 3 and 4 and is slightly above the exponential-dilution response.

If the reverse of Eq. (16a) is satisfied, the exponential in (19) may be expanded, with the result

$$
r_{\mathrm{pf}} \rightarrow \frac{k_{E} V X_{R}[M]^{2}}{(2+Z+1 / Z)\left[P_{D}\right]\left(1+k_{q}[M] / k_{f}\right)},
$$


and, if the reverse of Eq. (16b) also holds, the low-pressure limit is identical to Eq. (17). Thus the plug-flow and exponential dilution curves differ in Figs. 2-4 ony in the intermediate region between the high- and low-pressure extremes.

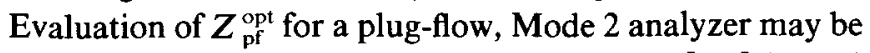
carried out by replacing $F_{M}[M]$ in Eq. (19) by $F_{D}\left[P_{D}\right](1+Z)$ from molecular conservation [Eq. (10)] and then differentiating with respect to $Z$.

\section{Liquid-phase chemiluminescent analyzer}

Many liquid-phase CL reactions are more complicated than the pseudo-first-order mechanism normally applicable to gas-phase CL analyzers. However, for liquid-phase CL reactions in a flow analyzer which follow processes (1-4) presented above, the response equations developed here apply with the significant simplification $[M]=\left[P_{D}\right]=\left[P_{R}\right]$. Thus these quantities may be cancelled where appropriate from the equations. Optimization of the analyzer still includes choice of the proper value of $Z$ as described by the $Z$ opt equations and the increase of $V$ or $X_{R}$ to reach the plateau region.

\section{DISCUSSION}

Some examples of Mode 1 and 2 behavior can be found in the gas-phase CL literature. Ridley and Howlett, ${ }^{10}$ studying the $\mathrm{NO} / \mathrm{O}_{3} \mathrm{CL}$ system in the laboratory, performed a Mode 2 experiment and observed peak response at an unstated pressure below ambient. Under balloon-borne conditions, however, their instrument would have corresponded with Mode 1, using fixed reagent flow rather than constant $Z$. Steffenson and Stedman 11 varied inlet flow, chamber volume, and outlet flow in the same chemical system. Here they first demonstrated the Mode 1 plateau, although their curves are distorted by variation of $F_{M}$ with $[M]$ due to flow restrictions in their outlet plumbing. These authors also distinguished the need for Mode 2 operation, given limited sample. McClenny et al. ${ }^{13}$ did Mode 1 experiments on the ozone/ ethylene and ozone/vinylchloride CL systems at 1-atm chamber pressure. Their fixed reagent flow caused a falloff in response at high sample flow rates, especially of the slowerreacting vinylchloride, but they would have observed the Mode 1 plateau if reagent flow had been larger, or had been adjusted for peak response at each sample-stream total flow rate. Kelly et al. ${ }^{25}$ optimized a commercial ozone CL analyzer for $\mathrm{CL}$ detection of reduced sulfur compounds. They found a response maximum occurred when $F_{D}=F_{R}=100$ $\mathrm{cc} / \mathrm{s}$ (i.e., $Z=1$ ), and [M] was slightly under 1 atm. However, they give insufficient details for us to judge the analyzer's operational mode. We have tested the ozone/ethylene CL system for conformance with Mode 1 and 2 behavior and have discussed Mode 3 operation of the $\mathrm{NO} / \mathrm{O}_{3} \mathrm{CL}$ analyzer. ${ }^{26}$

Sample tube transit time, signal rise time, and their resultant, the total instrumental response time, are important in some applications. If sample lines are required, short transit time can be obtained with low $[M]$ if the pressure reduction from ambient pressure occurs at the inlet to the plumbing. Also, at lower sample line pressures, any shift from photostationary equilibrium will be retarded. Furthermore, high $[M]$ may hinder complete mixing of the sample and reagent streams in the chamber. Thus increasing $V$ is preferable to increasing $[M]$ if short transit time, sensitivity, and plateau response are desired, at the cost of increased signal rise time.

In some CL chemical systems it may be desirable to use a pressure pump rather than a vacuum pump to feed the chamber, since the plateau response for slow reactions in practical chamber volumes may lie above atmospheric pressure. Difficulties would include perturbation of the detection gas by the pump itself, and deterioration of response times.

Many CL analyzers are operated at reduced chamber pressures. The above treatment shows that unless $F_{D}$ must be limited for some reason, no increase in response can result from low $[M]$ operation. However, there is a potential advantage of low $[M]$ besides those associated with reduced response time. If interfering chemiluminescence is produced by the reaction of the reagent $R$ with molecules other than $D$ in the sample, then it is possible to discriminate against this unwanted CL if the producing reaction is slow kinetically. Equation (17) indicates that at low [M] the response is proportional to $k_{E}$, so a low concentration of a reactive species may be detected even in the presence of a higher concentration of another, more slowly reacting species. The ability to discriminate against a slow reaction is shown by the twoheaded arrow in Fig. 4. Although the responses due to these two rate constants are the same when $[M]=1 \mathrm{~atm}$, reducing the chamber pressure to 0.001 atm suppresses the slow reaction relative to the fast one by a factor about equal to the rate constant ratio, here $10^{6}$.

It is not necessary to operate the chamber at ambient temperature, and a knowledge of the activation energies of the relevant chemical reactions will allow the use of Eq. (13) or its limiting variations to predict the advantages of either an increase or decrease in temperature. In the limiting response [Eq. (15)] the term $Y$ may have the strongest temperature dependence, and the merits of increasing or decreasing chamber temperature depend upon the relative activation energies of $k_{E}$ and $k_{L}$. Temperature manipulation has been discussed and employed by Ridley and Howlett ${ }^{10}$ and others. The condition for reaching the plateau, Eq. (16), is also temperature dependent, through the rate constants. Failure to at least maintain constant chamber temperature (e.g., with altitude) may lead to variations in instrumental response.

\section{CONCLUSIONS}

The equations developed here indicate the proper choice of relative flow rates into a CL analyzer, and the considerations in choosing the best values for the absolute flow rates, or the optimum pressure in the chamber. If sample and reagent gas are unlimited, then $F_{M}$ should be as large as the pump capacity will allow, and $F_{D}$ and $F_{R}$ should be adjusted to their optimum ratio in accordance with Eq. (14) or (20). The plateau of maximum response can be reached by ensuring that Eq. (16) is satisfied by variation of $V, X_{R}$, and [M]. 
This equation applies to both plug-flow and exponential-dilution analyzers.

At high chamber pressures the analyzer measures fractional rather than absolute concentration, an interesting feature for airborne sampling. If ambient pressure, $\left[P_{D}\right]$, is subject to variation during sampling, then the linear response in mole fraction $X_{D}$ may be assured by seeing that $F_{M}$ remains constant and that the plateau behavior is maintained. If desired, $F_{D}$ and $V X_{R}$ may be selected from a crossover region where the analyzer responds linearly to ambient concentration. No response increase can be obtained by operating the chamber under reduced pressure in these circumstances. However, if total sample volume precludes large sampling rates then Eq. (18) may be used to decide upon an optimum pressure. One must be aware, however, that the detector may be operating in a pressure-sensitive region where the response to absolute concentration or mole fraction at changing ambient pressure is complicated. In cases where interfering CL reactions are occurring, chamber pressure variation may allow discrimination against the interferences. If reagent is limited, and/or insensitivity to ambient pressure is needed, it may be desirable to operate the analyzer under Mode 3 conditions.

Generally, large reactor volumes are preferred in order to maximize response. Thus the exponential-dilution chamber considered in greatest detail may be more realistic than the plug-flow chamber. However, any well-designed reactor should fall between these two extremes, which are seen in Figs. 2-4 to give similar responses, so it is only necessary to see that the two flows are well-mixed throughout the chamber volume and that the walls are highly reflective.

The general principles derived here may be applied to improvement of the detection limits and accuracy of present analyzers and to the search for new chemiluminescent reactions. For cases where the necessary rate constants are unknown, these principles may be used either to measure their values ${ }^{27}$ or to carry out an efficient empirical optimization.

\section{ACKNOWLEDGMENTS}

This work was supported, in part, by N. S. F. Atmospheric Chemistry Program grant ATM 8003312, U. S. E. P. A. Office of Research and Development grant R807733, and the Portland State University Research and Publications Committee. Although the research described in this article was funded in part by the U.S.E.P.A., it has not been subjected to the Agency's required peer and administrative review and, therefore, does not necessarily reflect the view of the Agency and no official endorsement should be inferred.

'P. N.Clough and B. A. Thrush, Trans. Faraday Soc. 63, 915 (1967)

${ }^{2}$ B. A. Thrush, Chem. Br. 2, $287(1966)$.

${ }^{3}$ B. J. Finlayson, J. N. Pitts, Jr., and R. Atkinson, J.Am. Chem. Soc. 96, $5356(1974)$.

${ }^{4}$ G. W. Nederbragt, A. van der House, and J. van Duijn, Nature 206, 87 (1965).

${ }^{5}$ J. A. Hodgeson, K. J. Krost, A. E. O'Keefe, and R. K. Stevens, Anal. Chem. 42, 143 (1970).

${ }^{6}$ A. Fontijn, A. J. Sabadell, and R. J. Ronce, Anal. Chem. 42, 575 (1975).

${ }^{7}$ R. K. Stevens and A. E. O'Keefe, Anal. Chem. 42, 143 (1970).

${ }^{8}$ F. J. Warren and F. Babcock, Rev. Sci. Instrum. 41, 280 (1970).

'D. J. Stedman, E. E. Daby, F. Stuhl, and H. Niki, J. Air Poll. Control Assoc. 22, $260(1972)$

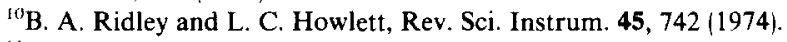

${ }^{11}$ D. M. Steffenson and D. H. Stedman, Anal. Chem. 46, 1704 (1974).

${ }^{12}$ A. Fontijn and R. Ellison, Environ. Sci. Technol. 9, 1157 (1975).

${ }^{13}$ W. A. McClenny, B. E. Martin, R. E. Baumgardner, Jr., R. K. Stevens and A. E. O'Keefe, Environ. Sci. Technol. 10, 810 (1976).

${ }^{14}$ T. J. Kelly, D. H. Stedman, J. A. Ritter, and R. B. Harvey, J. Geophys. Res. 85, 7417 (1980).

${ }^{15}$ C. R. Roy, I. E. Galbally, and B. A. Ridley, Quart. J. Roy. Met. Soc. 106, $887(1980)$.

${ }^{16}$ D. Kley and M. McFarland, Atmos. Technol. 12, 63 (1980).

${ }^{17}$ A. Fontijn, J. N. Volltrauer, and W. R. Frenchu, Environ. Sci. Technol. 14, $324(1980)$.

${ }^{15}$ D. Kley, J. W. Drummond, M. McFarland, and S. C. Liu, J. Geophys. Res. 86, 3153 (1981).

${ }^{19} \mathrm{P}$. M. Houpt and F. Langeweg, Anal. Chim. Acta 124, 15 (1981).

${ }^{20} \mathrm{~K}$. Fujiwara, Y. Watanabe, K. Fuwa, and J. D. Winefordner, Anal. Chem. 54, 125 (1982).

${ }^{21}$ B. A. Ridley, Atmos. Technol. 9, 35 (1978).

${ }^{22}$ W. A. Kummer, J. N. Pitts, Jr., and R. P. Steer, Environ. Sci. Technol. 10. $1045(1971)$

${ }^{23}$ J. N. Pitts, Jr., B. J. Finlayson, H. Akimoto, W. A. Kummer, and R. P. Steer, Adv. Chem. Ser. 113, 246 (1972).

${ }^{24}$ T. J. Kelly, J. S. Gaffney, M. F. Phillips, and R. L. Tanner, Anal. Chem. 55, $138(1983)$.

${ }^{25}$ A. Fontijn, Modern Fluorescence Spectroscopy, edited by E. L. Wehry (Plenum, New York, 1976), pp. 159-189.

${ }^{26}$ A. A. Mehrabzadeh, R. J. O'Brien, and T.M. Hard, Anal. Chem. 55, 1660 (1983).

${ }^{27}$ A. A. Mehrabzadeh, T. M.Hard, and R. J. O'Brien, "Rate Constants for the Ozone/Ethylene Chemiluminescent Reaction" (in preparation). 